\title{
ABORDAGEM SOBRE O TDAH: CLASSIFICAÇÃO, FALSO DIAGNÓSTICO E IMPACTOS DO TRATAMENTO MEDICAMENTOSO EM CRIANÇAS
}

\author{
S.A.M.S. ALVES ${ }^{2}$, A.S. FERREIRA², A.C. SILVA², R.S. OLIVEIRA ${ }^{1}$, E.O. SILVA ${ }^{1}$, \\ I.N.G. BARBOSA ${ }^{1}$, M.C.T.B. ARAÚJO ${ }^{2}$, R.M.F. SILVA', P.J. ROLIM NETO ${ }^{1}$ \\ ${ }^{1}$ Universidade Federal de Pernambuco, Centro de Ciências da Saúde, Departamento de \\ Ciências Farmacêutica \\ ${ }^{2}$ Centro Universitário Maurício de Nassau, Departamento de Ciências Farmacêuticas \\ E-mail para contato: oliveira.silva.rodrigo19@gmail.com
}

\begin{abstract}
RESUMO - O transtorno de déficit de atenção com hiperatividade (TDAH) é um distúrbio neuropsicomotor de cunho comportamental, relacionado com fatores de atenção $e$ desenvolvimento humano. Sua classificação é definida de acordo com critérios clínicos, embora não haja consenso sobre a segurança em relação ao diagnóstico. Medicamentos são prescritos para tratamento pacientes confirmados, nos últimos anos observou-se um aumento no consumo desses medicamentos principalmente por crianças e jovens. O objetivo do presente trabalho foi descrever a classificação dos tipos de TDAH, avaliando a segurança, diagnostico e as conseqüencias do tratamento medicamentoso de pacientes que não teriam real necessidade. A metodologia baseou-se em um estudo observacional com corte transversal de 2009 a 2015, utilizando legislações e periódicos obtidos das bases de dados: Scielo, Pubmed e Bireme. A classificação atual não é tida como segura por parte da comunidade cientifica que estuda os transtornos de atenção, o que caracteriza os erros de diagnóstico e consequente consumo de anfetaminas por um público bastante jovem, refletindo em problemas futuros devido aos efeitos adversos associados. Conclui-se que há a necessidade de desenvolver uma classificação mais segura e a partir daí um diagnóstico adequado baseado também em exames complementares e baseados em informações de uma equipe multidisciplinar, a fim de evitar erros na prescrição e consumo de drogas que afetam consideravelmente crianças e jovens.
\end{abstract}

Palavras-chave: TDHA, metilfenidato, tratamento medicamentoso.

ABSTRACT - Attention deficit hyperactivity disorder (ADHD) is a behavioral neuropsychomotor disorder related to attention and human development factors. Its classification is defined according to clinical criteria, although there is no consensus about safety in relation to the diagnosis. Medications are prescribed to treat confirmed patients, in recent years there has been an increase in the consumption of these drugs mainly by children and young people. The aim of the present study was to describe the classification of ADHD types, evaluating the diagnostic safety and the consequences of the drug treatment of patients who would not have a real need. The methodology was based on a cross-sectional observational study from 2009 to 2015, using legislations and periodicals obtained from the databases: Scielo, Pubmed and Bireme. The current classification is not considered safe by the scientific community studying attention 
disorders, which characterizes the errors of diagnosis and consequent consumption of amphetamines by a very young public, reflecting in future problems due to the associated adverse effects. It is concluded that there is a need to develop a safer classification and hence an adequate diagnosis based also on complementary exams and based on information from a multidisciplinary team, in order to avoid errors in the prescription and consumption of drugs that considerably affect children and young.

Key words: $A D H D$, methylphenidate, drug therapy.

\section{INTRODUÇÃO}

O transtorno de déficit de atenção e hiperatividade (TDAH) é uma doença neuropsicomotora que acomete pessoas de ambos os sexos, diagnosticada inicialmente na infância, com idade entre 3 e 5 anos. A fase escolar é a que melhor apresenta os sinais de comportamento e aprendizado, pois normalmente se verificam crianças agitadas, isoladas, desatentas, com dificuldades de socialização e que não são capazes de finalizar tarefas comuns a crianças da mesma idade (SENO, 2010).

As dificuldades em realização do diagnóstico se dão pela falta de clareza dos métodos de classificação, existindo a necessidade de ser feito em conjunto com outros exames e avaliações adicionais, além de ter o envolvimento de outros profissionais. A participação da família e da escola são essenciais na observação desses comportamentos e podem ser o ponta pé inicial para o encaminhamento para o serviço de saúde. Embora inicialmente haja resistência por parte dos pais em relação ao tratamento, a falta de informação e a credibilidade social que se tem na figura do profissional médico são questões que determinam a adesão ao tratamento destas crianças, que farão uso de medicamentos psicotrópicos (BRZOZOWSKI, 2009).

A Ritalina (Metilfenidato) é o medicamento de primeira escolha no tratamento do TDAH. Na década de 90 houve publicações a respeito da produção deste medicamento, que chegou a um aumento de $700 \%$ nos Estados Unidos, devido ao numero de crianças sob o uso da medicação. O aumento de $85 \%$ do consumo é voltado principalmente para o tratamento da desordem da atenção (CALIMAN, 2009).

O objetivo é mostrar como a realização de tratamento medicamentoso feito a partir de erros de diagnóstico pode interferir na saúde de crianças e jovens com suposta TDAH e suas consequências futuras.

\section{MATERIAIS E MÉTODOS}

A metodologia baseou-se em um estudo observacional com corte transversal de 2009 a 2015, utilizando legislações e periódicos obtidos das bases de dados: Scielo, Pubmed e Bireme.

\section{RESULTADOS E DISCUSSÃO}

Segundo Seno (2010) existe quatro tipos de classificação para a identificação do perfil de crianças com TDAH, como descrita no quadro 1. 
Quadro 1 - Classificação para identificação do TDAH.

\begin{tabular}{|l|l|}
\hline \multicolumn{1}{|c|}{ Tipos } & \multicolumn{1}{|c|}{ Características } \\
\hline Desatento & $\begin{array}{l}\text { Não enxerga detalhes, faz erros por falta de cuidado, apresenta } \\
\text { dificuldade em manter a atenção, parece não ouvir, tem dificuldade em } \\
\text { seguir instruções, desorganização, evita/não gosta de tarefas que } \\
\text { exigem um esforço mental prolongado, distrai-se com facilidade, } \\
\text { esquece atividades diárias. }\end{array}$ \\
\hline $\begin{array}{l}\text { Hiperativo/ } \\
\text { Impulsivo }\end{array}$ & $\begin{array}{l}\text { Inquietação, mexer as mãos e os pés, remexer-se na cadeira, dificuldade } \\
\text { em permanecer sentada, corre sem destino, sobe nos móveis ou muros, } \\
\text { dificuldade em engajar-se numa atividade silenciosamente, fala } \\
\text { excessivamente, responde perguntas antes delas serem formuladas, } \\
\text { interrompem assuntos que estão sendo discutidos e se intrometem nas } \\
\text { conversas. }\end{array}$ \\
\hline Combinado & $\begin{array}{l}\text { Quando o indivíduo apresenta os dois conjuntos de critérios desatento e } \\
\text { hiperativo/impulsivo. }\end{array}$ \\
\hline $\begin{array}{l}\text { Não } \\
\text { específico }\end{array}$ & $\begin{array}{l}\text { Quando as características apresentadas são insuficientes para se chegar } \\
\text { a um diagnóstico completo, apesar dos sintomas desequilibrarem a } \\
\text { rotina diária. }\end{array}$ \\
\hline
\end{tabular}

Fonte: SENO, 2010 (adaptado)

Esta classificação ajuda na confirmação e definição do tipo de distúrbio do indivíduo investigado, mesmo assim pode haver controvérsias, pois em se tratando de um transtorno neurobiológico e multifatorial, outros aspectos como comportamento social e capacidade de desenvolvimento são características subjetivas que trazem discrepâncias no resultado dos diagnósticos.

Os estudos de Seno (2010) e Larroca (2012) mostraram que ainda não existe um modelo definitivo que determine a classificação dos transtornos de atenção, seguido pelo de Calimam (2009) que aponta o comportamento antissocial como característico desse distúrbio ou doença, esclarecendo que há duvida quanto ao enquadramento de pacientes que são fisiologicamente normais, mas que não acompanham o contexto social, político e econômico dito como "normal" nos padrões atuais.

A ausência do perfil social, pode levar a uma falsa percepção de TDAH, o que gera consequente adesão de tratamento medicamentoso realizado com a Ritalina. A Ritalina é um psicoestimulante, do grupo das anfetamina, seu mecanismo de ação ainda não é bem elucidado mas tem-se a hipótese de ser um bloqueador de dopamina. Inicialmente era indicada para cansaço em idosos e fadiga em pacientes psiquiátricos e hoje para o tratamento de transtorno de déficit de atenção/hiperatividade, narcolepsia e obesidade. Seus efeitos colaterais são observados a curto, médio e longo prazo conforme se pode observar no quadro 2 (ORTEGA et.al., 2010). 
Quadro 2 - Efeitos adversos da utilização de Ritalina por pacientes com diagnóstico do TDAH.

\begin{tabular}{|l|l|}
\hline \multicolumn{1}{|c|}{ Prazo } & \multicolumn{1}{c|}{ Efeitos Adversos } \\
\hline $\begin{array}{l}\text { Curto } \\
\text { prazo }\end{array}$ & $\begin{array}{l}\text { Insônia, redução do apetite, anorexia, ansiedade, irritabilidade, labilidade } \\
\text { emocional, podendo ainda provocar cefaléia e dor abdominal. }\end{array}$ \\
\hline $\begin{array}{l}\text { Médio } \\
\text { prazo }\end{array}$ & $\begin{array}{l}\text { Alterações de humor, tiques, pesadelos, isolamento social e raramente, } \\
\text { psicose. }\end{array}$ \\
\hline $\begin{array}{l}\text { Longo } \\
\text { Prazo }\end{array}$ & $\begin{array}{l}\text { Redução da estatura, dependência química e psicológica e efeitos } \\
\text { cardiovasculares. }\end{array}$ \\
\hline
\end{tabular}

Fonte: MARTINS et.al. 2015 (adaptado)

Outro ponto a ser observado, relaciona-se a dose, pois estpa deve ser empregada de acordo com o perfil e idade do paciente, uma vez ela que pode interferir diretamente no resultado do tratamento e no aparecimento de reações adversas.

$\mathrm{O}$ aumento dos diagnósticos precedidos de tratamento medicamentoso com drogas psicotrópicas traz um alerta ao aumento do consumo do metilfenidato, principalmente para aqueles que foram classificados como portadores do transtorno sem tê-lo, levantando a necessidade de mais publicações referentes ao tema e poder assim contribuir com um maior esclarecimento sobre a doença e as consequências errôneas da profilaxia medicamentosa nestes pacientes.

\section{CONCLUSÃO}

Conclui-se que há necessidade de uma classificação mais completa sobre o diagnóstico do transtorno de déficit de atenção/hiperatividade e de divulgação de mais informações para as pessoas sobre este assunto tem relevância global. Evitando erros que vão desde o diagnóstico ate prescrição de medicamentos e os ajustes das doses, que podem ser extremamente prejudiciais para os pacientes pediátricos. Principalmente se esses pacientes forem saudáveis e fizerem uso indevido, podendo comprometer a qualidade de vida da criança ou do adolescente.

\section{REFERÊNCIAS}

BRZOZOWSKI, fs; CAPONI, S. Transtorno de Déficit 1165 de Atenção com Hiperatividade: classificação e classificados1. Rev. de Saúde Coletiva, v. 19, p. 11651187, 2009.

CALIMAM, LV. A constituição sócio-médica do "fato TDAH". Psicologia \& Sociedade, v. 21, p. 135-144, 2009.

LARROCA, LM; DOMINGOS, NM. TDAH - Investigação dos critérios para diagnóstico do subtipo predominantemente desatento. Rev. Sem. da Assoc. Bras.de Psicol. Esc.e Educ., v. 16, p. 113-123, 2012

MARTINS, FAG et.al. Metilfenidato em Crianças no Brasil: Análise Crítica de Publicações Científicas de 2004 a 2014. Rev. Neurocienc., v. 23, p. 190-204, 2015

ORTEGA, F et.al. A ritalina no Brasil: produções, discursos e práticas. Interface Comunic., Saude, Educ., v. 14, p. 499-510, 2010.

SENO, MP. Transtorno do Déficit de Atenção e Hiperatividade (TDA H): o que os educadores sabem. Rev. Psicopedagogia, v. 27, p. 334-43, 2010. 